\title{
Effect of structural modification at the 4, 3', and 2' positions of doxorubicin on topoisomerase II poisoning, apoptosis, and cytotoxicity in human melanoma cells
}

\author{
Beata M. Gruber ${ }^{1}$, Elżbieta L. Anuszewska ${ }^{1}$ Irena Bubko ${ }^{1}$, Aneta Goździk \\ Izabela Fokt ${ }^{2}$ and Waldemar Priebe ${ }^{2}$
}

1 Department of Biochemistry and Biopharmaceuticals, National Institute of Medicines, Warsaw, Poland

${ }^{2}$ Department of Experimental Therapeutics, M.D. Anderson Cancer Center, Houston, TX 77030, USA

Received: 2007.01.23, Accepted: 2007.02.28, Published online first: 2007.06.08

\begin{abstract}
Introduction: The mechanism of the cytotoxicity of anthracyclines is pleiotropic and its significance in cell growth inhibition seems to be highly specific and dependent on cell type and anthracycline drug. Resistance and the high cardiotoxicity of anthracyclines have stimulated many studies aimed at identifying critical substituents required for optimal activity. Many authors point to the fact that the double-strand breaks, the consequence of the activity of topoisomerase II poisons, and the inability of cells to repair the DNA lesions are the signal for apoptosis. The aim of this study was to define the influence of 4-demetoxy 2'-halogenated analogs with altered basicity at the 3'-position on topoisomerase II and the relationship of that interaction with apoptosis and the cytotoxicity of these novel anthracyclines. Parental human ME18 melanoma cells and the ME18/R subline, obtained experimentally, resistant to doxorubicin (DOX), exposed to 1.7 and $8.6 \mu \mathrm{M}$ DOX or its analogs, annamycin and WP903 (both 0.3 and $3.0 \mu \mathrm{M}$ ) were studied.

Materials and Methods: The MTT test was used to assay cytotoxicity. Interaction of the drugs with topoisomerase II and apoptosis were done by Western blot and fluorescence microscopy using Hoechst 33342.

Results: The structural changes at positions 4, 2', and 3' can influence topoisomerase II interaction and apoptotic activity, although correlation between these events and cytotoxic consequences has not been proved.

Conclusions: The biological response of the cells to the structurally similar anthracyclines may be variable and probably depends on the cell type which seems to be an additional problem in the multifactorial resistance of tumor cells to anthracyclines.
\end{abstract}

Key words: apoptosis, anthracyclines, topoisomerase II poisons, cytotoxicity.

Corresponding author: Beata M. Gruber, Department of Biochemistry and Biopharmaceuticals, National Institute of Medicines, Chełmska 30/34, 00-725 Warsaw, Poland, tel.: +48 22 841-21-65, fax: +48 22 841-06-52, e-mail: b-gruber@il.waw.pl

\section{INTRODUCTION}

The use of anthracyclines as antitumoral drugs dates back to late 1960s and early 1970s. They are used in the treatment of solid tumors, including breast cancer, tumors of the bile ducts, uterus, esophagus, liver, osteosarcomas, soft tissue sarcomas, as well as acute myeloblastic leukemia [11]. The mechanism of the cytotoxicity of of anthracycline is pleiotropic and its significance in cell growth inhibition seems to be highly specific, depending on cell type and the anthracycline drug. It is still unknown which route of action is predominant for drug resistance, which is a large obstacle in anthracycline therapy. The most understood mechanism is that related to transmembrane proteins, e.g. P-gp 170 or MRP-1 or -2 overexpression, which results in the decreased intracellular drug accumulation observed in resistant cells. Also considered to be associated with the development of drug resistance are changes in drug metabolism, increased activity of detoxifying enzymes such as glutathione transferase, peroxidase, and superoxide dismutase, alteration in topoisomerase II protein levels, and DNA cleavage of that enzyme [22].

Topoisomerase II poisons such as doxorubicin (DOX) or daunorubicin trap the cleavable complexes 
topoisomerase II-DNA and inhibit the resealing of DNA breaks created by the enzyme. So-called "catalytic inhibitors", such as aclarubicin, inhibit the enzyme's binding to its DNA substrate or lock the homodimeric topoisomerase II in the form of a closed bracelet surrounding DNA at the post-religation step, like bisdioxopiperazine. But only topoisomerase II poisons can generate DNA breaks [25]. The "topo II poisons" are reported to be potentially more important and possibly relevant mechanisms of resistance in cells that may overexpress P-gp [12].

Many authors point to the fact that the doublestrand breaks, a consequence of the activity of topoisomerase II poisons, and the inability of the cells to repair the DNA lesions are the signal for apoptosis $[6,8,18,19$, 24]. On the basis of literature data it can be said that tumors with higher apoptosis and topoisomerase II $\alpha$ overexpression tend to be more responsive to anthracyclines $[2,20]$. A number of studies have shown that the profiles of topoisomerase II protein or gene expression affect the response to anthracyclines [9, 16, 17]. The observed link between topoisomerase II and clinical response to anthracyclines-based chemotherapy implies that topoisomerase II deserves further testing in a prospective setting as a predictive marker $[4,21]$.

Although the topoisomerase II inhibitors have a common intracellular target, the molecular basis of their variable therapeutic efficacy is still not fully understood [3]. Resistance and the multifactorial mechanism of the cytotoxic action as well as the high cardiotoxicity of anthracyclines have stimulated many studies aimed at identifying critical substituents required for optimal activity and, consequently, new anthracycline analogs having better therapeutic index are being sought [3].

It was noted that topoisomerase II targeting or interaction with P-gp is related to the removal of a 4-metoxy group and alteration of basicity at the 3' position in the DOX molecule $[23,26]$. On the basis of a number of studies it can be said that chemical modification makes it possible to "select" mechanisms, for example to reduce drug-P-gp interaction but maintain or increase the desired cytotoxic action. In other words it is now possible to design such "molecular switches" to influence their antitumoral efficacy.

As part of the collaboration with Prof. Waldemar Priebe's group at the University of Texas M.D. Anderson Cancer Center, Houston, Texas, USA, the anthracycline derivatives WP903 with Annamycin (ANN), currently being evaluated in clinics in leukemia patients, were chosen in screening studies as new promising anticancer drugs (Fig.1). The aim of this study was to define the influence of 4-demetoxy 2'-halogenated analogs with altered basicity at the 3 ' position on topoisomerase II and the relationship of that interaction with apoptosis and the cytotoxicity of these novel anthracyclines. The stabilization of the complexes was studied with Western blot (band depletion assay). Apoptotic events were evaluated morphologically and in PARP cleavage assay.<smiles>Cc1cccc2c1C(=O)c1c(O)c3c(c(O)c1C2=O)C[C@@](O)(C(=O)CO)C[C@@H]3OC1CC2CCCC(C2)C1N</smiles>

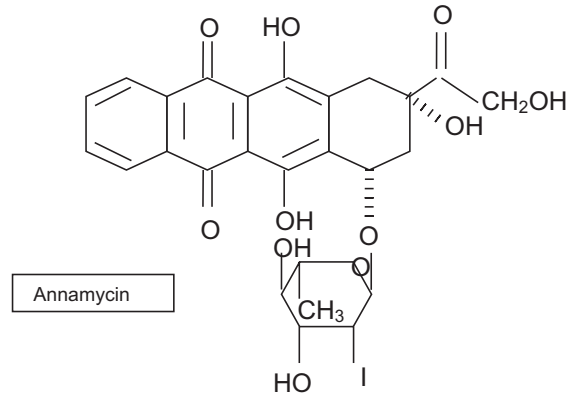

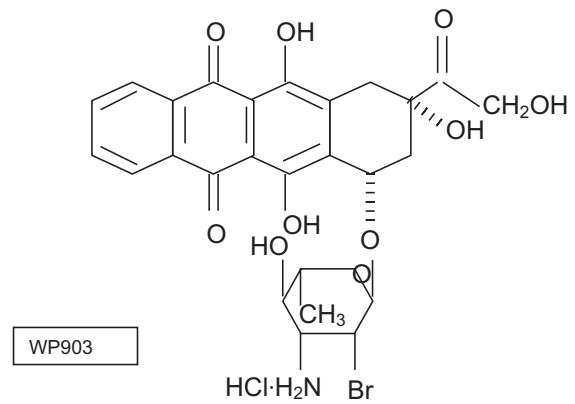

Fig. 1. Structures of DOX and its analogs, ANN and WP903.

\section{MATERIALS AND METHODS}

Drugs

DOX was purchased from Fluka (Germany), and WP903 and ANN were received from the University of Texas M.D. Anderson Cancer Center, Houston, TX, USA.

Cells

The ME18 human melanoma cell line was obtained from the M. Skłodowska-Curie Memorial Cancer Center and Institute of Oncology, Warsaw, Poland. ME18/R, an $m d r$-1-expressing subline resistant to DOX, vinblastine, and methotrexate, was obtained experimentally in our laboratory [1].

The cells were grown in Minimum Essential Medium (MEM) supplemented with $10 \%$ fetal calf serum and antibiotics (penicillin $10.000 \mathrm{u} / \mathrm{ml}$, streptomycin $10 \mathrm{mg} / \mathrm{ml}$, amphotericin B, 0.9\%). The ME18/R cells were maintained in MEM with DOX $0.04 \mathrm{uM}$.

All cell cultures were mycoplasma free. 
MTT test

The test was performed as described in our previous study [13]. The final concentrations of DOX, ANN, and WP903 ranged from 0.09 to $10 \mu \mathrm{M}$. The cells were exposed to the drugs for $48 \mathrm{~h}$. In the further studies, all the cell lines tested were exposed to the same drug concentrations.

\section{Morphological evaluation}

Twenty-four-hour cell cultures were exposed for 24 $\mathrm{h}$ to the drugs used at the concentrations of 1.7 and 8.6 $\mu \mathrm{M}$ DOX or 0.3 and $3.0 \mu \mathrm{M}$ ANN or WP903. Apoptotic symptoms such as chromatin condensation and apoptotic bodies were observed in a fluorescence microscope using Hoechst 33342. The procedure was described in detail earlier [13].

\section{Band depletion assay and apoptosis detection}

Twenty-four-hour cell cultures were exposed for 24 $\mathrm{h}$ to the drugs tested used at the above concentrations. Nuclear extract preparation and Western blot analysis were performed as previously described [13]. The bands obtained were quantified using the Gel Doc 2000 System by BioRad with Quantity One 4.4.0 1-D Analysis Software. For gel quantity evaluation, the bands of interest were normalized to $\beta$-actin.

\section{Statistical analysis}

The statistical evaluation of the results was performed using Student's $t$-test and the Cochrane Cox test for unrelated samples.

\section{RESULTS AND DISCUSSION}

The results obtained in this study indicate a lack of correlation between the stabilization of cleavable complexes, induction of apoptosis, and the cytotoxic activity of the tested anthracyclines. DOX, the least cytotoxic among the tested drugs (Table 1), stabilized the cleavable complexes in ME18 cells similarly to ANN, but these comparable effects required an over twofold higher concentration of DOX (Fig. 2a, b). However, apoptosis, observed as PARP cleavage (Fig. 3a, b) and visible through morphological symptoms (data not shown) occurred only in ME18 cells exposed to ANN. None of these effects were noted in ME18/R cells regardless of the drug used.

WP903 was shown to be an apoptotic inducer of ME18 and ME18/R cells (Fig. 3c). This was also confirmed in the microscopic analysis (data not shown). Annexin V-FITC and the TUNEL tests yielded the same results as those given in our earlier study [14]. That analog was noted as the most active cytotoxic drug towards ME18/R cells (Table 1). In the light of the
Table 1. $\mathrm{IC}_{50}$ values determined by the MTT test

\begin{tabular}{lccr}
\hline Anthracycline & ME18 & ME18/R & $\mathrm{RF}^{\mathrm{d}}$ \\
\hline DOX & $12.21 \pm 3.18^{\mathrm{a}}$ & $48.68 \pm 2.06$ & 4.0 \\
ANN & $0.90 \pm 0.17^{\mathrm{a}, \mathrm{b}}$ & $44.15 \pm 2.26$ & 49.0 \\
WP903 & $1.48 \pm 0.41^{\mathrm{a}, \mathrm{b}}$ & $5.41 \pm 0.43^{\mathrm{c}}$ & 3.7 \\
\hline
\end{tabular}

The cells were treated with the drugs, each used in the range $0.09-10 \mu \mathrm{M}$ for $48 \mathrm{~h}$, then the MTT test was done as described earlier [13].

Results represent the mean \pm SEM $(n=8)$.

${ }^{\mathrm{a}} \mathrm{IC}_{50}$ values $(\mu \mathrm{M})$ statistically different from $\mathrm{IC}_{50}$ defined for DOX, ANN, or WP903 in ME18/R cells $(\mathrm{p}<0.05)$.

${ }^{\mathrm{b}} \mathrm{IC}_{50}$ values statistically different from $\mathrm{IC}_{50}$ defined for DOX in ME18 cells $(\mathrm{p}<0.05)$.

${ }^{\mathrm{c}} \mathrm{IC}_{50}$ value statistically different from $\mathrm{IC}_{50}$ defined for DOX or ANN in ME18/R cells $(\mathrm{p}<0.05)$.

${ }^{\mathrm{d}}$ Resistance factor $\left(\mathrm{IC}_{50 \mathrm{ME} 18 / \mathrm{R}} / / \mathrm{IC}_{50 \mathrm{ME} 18}\right)$.

study, the induction of apoptosis does not seem to be related to stabilization of cleavable complexes, as presented in Fig. 2c.

The results obtained in this study are confirmed by Gruber et al. [15] and Yamazaki et al. [27]. The authors noted that the difference in drug sensitivity to DOX might not be explainable by the topo II $\alpha$ levels. In turn, Ramachandran et al. [24] noted that low DOX sensitivity of the human melanoma cell lines FCCM-9 and NH was due to reduced DNA damage and low topo II activity. That dependence was also confirmed by De Graff et al. [7], who observed that the non-toxic topo II inhibitor WR-1065 protected Chinese hamster V79 cells against DOX-induced cytotoxicity.

The results obtained here indicate that the cytotoxic mechanisms of the anthracycline drug may be in part related to apoptosis induction and may also depend on the drug used. As shown, the stabilization of cleavable complexes is not a condition sine qua non for apoptosis induction. According to Li and Liu [19], the role of topo II in mediating apoptosis may depend on the kind of ligands participating in apoptotic induction. It is known that apoptosis, which is a carefully regulated process of cell death, may proceed through mechanisms which vary according to the cell type $[5,10]$.

As shown, ANN and WP903 are clearly different from DOX in evoking the stabilization of topoisomerase II complexes and apoptosis, which proves the importance of the structural changes at the 4, 3', and 2' positions of the anthracycline molecule in the biological activity of these drugs. The current results are in agreement with those of Priebe [23] and Trevino et al. [26], who indicated that the demetoxy analogs are more active in trapping topoisomerase II cleavable complexes.

It is interesting to note that the similar structural changes, i.e. reduced basicity at $3^{\prime}$ and the removal of the metoxy group at $\mathrm{C}_{4}$ of the aglycone as in ANN and WP903, are supposed to be important only for the cytotoxic activity of WP903, but do not matter in the case of ANN (Table 1). 
a
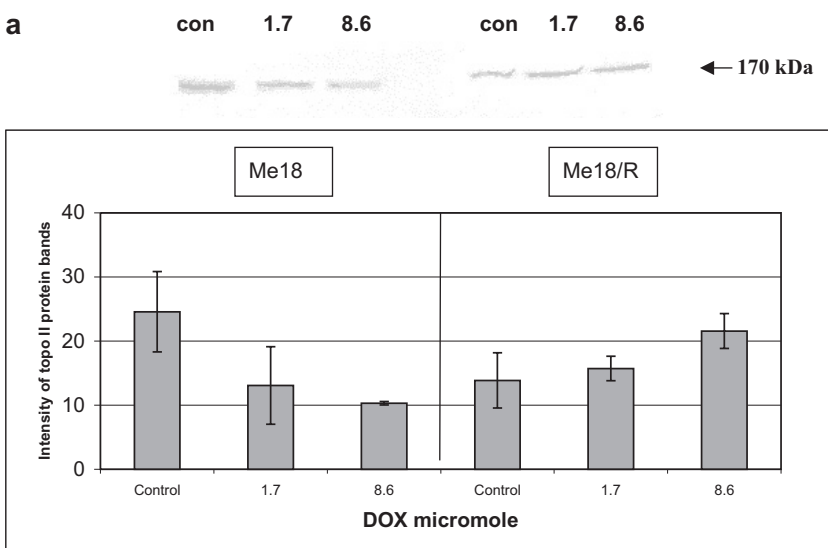

b

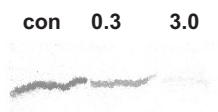

con $\quad 0.3 \quad 3.0$

$\longleftarrow 170 \mathrm{kDa}$

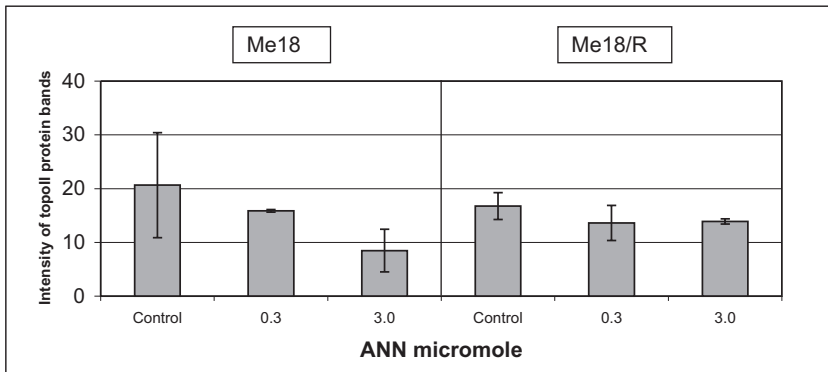

C
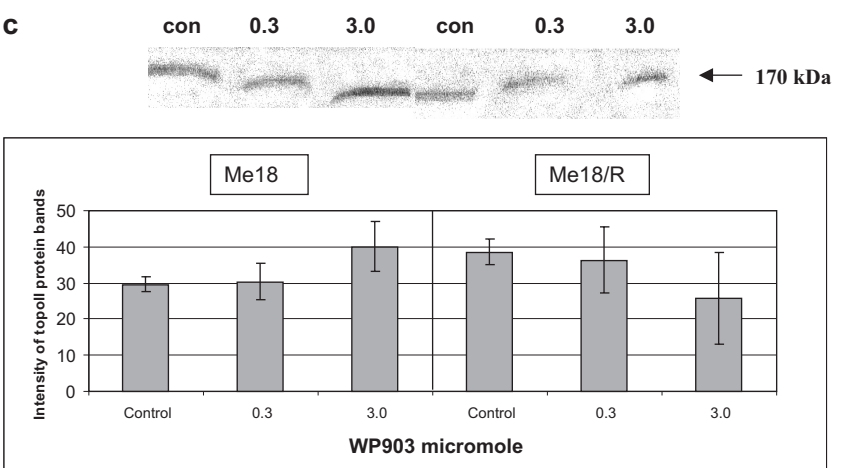

Fig. 2. Topoisomerase II $\alpha$ band depletion assay. 24-h cell cultures were treated for $24 \mathrm{~h}$ with: $\mathbf{a}-\mathrm{DOX}(1.7$ and $8.6 \mu \mathrm{M}), \mathbf{b}-\mathrm{ANN}$ $(0.3$ and $3.0 \mu \mathrm{M})$, or $\mathbf{c}-\mathrm{WP} 903(0.3 ; 3.0 \mu \mathrm{M})$. Western blot analysis was done with nuclear extracts according to the procedure given in our previous study [11]. Results represent the mean \pm SEM, n=3-4.

As Priebe [23] hypothesized, the 3'-amino group in the anthracycline analogs is an important structural element stabilizing the interaction of the drug with P-gp. Our earlier studies using verapamil, probenecid, and cyclosporine, the inhibitors of P-gp or MRP-1,2 transmembrane pumps, showed that these substances did not significantly affect the intracellular WP903 concentration in ME18/R cells measured fluorometrically as $\begin{array}{llllllll}\text { a } & \text { con } & 1.7 & 8.6 & \text { con } & 1.7 & 8.6\end{array}$
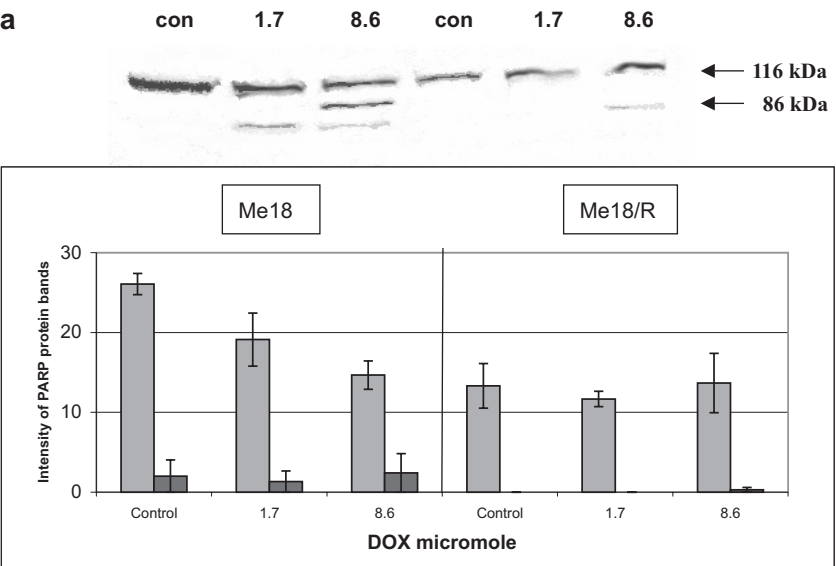

b
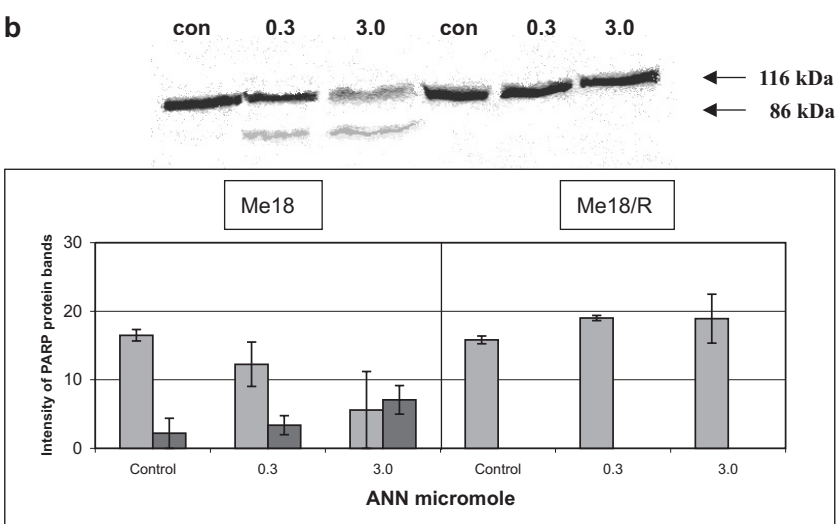

C
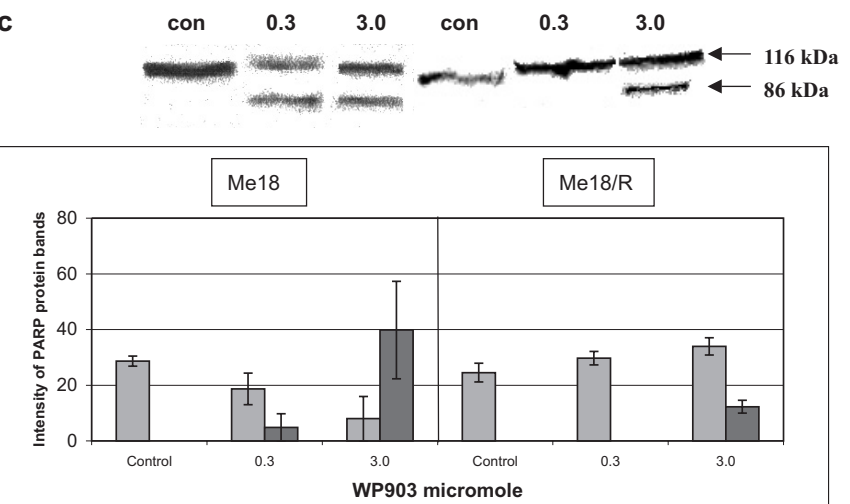

Fig. 3. PARP cleavage induced by DOX (a); ANN (b); WP903 (c). Light fields stand for PARP, $116 \mathrm{kDa}$; dark fields for PARP fragments, $86 \mathrm{kDa}$. 24-h cell cultures were exposed for $24 \mathrm{~h}$ to the drugs given in the concentrations: DOX (1.7 and 8.6 $\mu \mathrm{M})$, ANN and WP903 (both 0.3 and $3.0 \mu \mathrm{M}$ ). Western blot analysis was done with nuclear extracts according to the procedures described in our earlier study [11]. Results represent the mean \pm SEM, $n=3-4$.

$\mathrm{ng} / 10^{6}$ cells at different time-points during incubation with the drug or after removing medium with the drug compared with cells untreated with inhibitors (data not shown). The efflux of WP903 was observed at similar levels in cells untreated or treated with transmembrane protein inhibitors. This means that the increased cyto- 
toxicity of WP903 observed in ME18/R cells is probably connected not only with a greater affinity of that drug to the transmembrane proteins.

On the basis of these studies it can be said that the mechanisms of action of anthracyclines are cell dependent, which seems to be an additional problem in the multifactorial resistance of tumor cells to anthracyclines.

Acknowledgment: This work was supported in part by a grant from The Welch Foundation (Houston, Texas, USA) to W. Priebe and research grant No. 3PO5FO1722 from the State Committee for Scientific Research (KBN, Poland).

\section{REFERENCES}

1. Anuszewska E. L., Gruber B. M. and Koziorowska J. H. (1997): Studies on adaptation to adriamycin in cells pretreated with hydrogen peroxide. Biochem. Pharmacol., 54, 597-603.

2. Arpino G., Ciocca D. R., Weiss H., Allred D. C., Daguerre P., Vargas-Roig L., Leuzzi M., Gago F., Elledge R. and Mohsi S. K. (2005): Predictive value of apoptosis, proliferation, HER-2, and topoisomerase II alpha for anthracycline chemotherapy in locally advanced breast cancer. Breast Cancer Res. Treat., 92, 69-75.

3. Capranico G., Supino R., Binaschi M., Capolongo L., Grandi M., Suarato A. and Zunino F. (1994): Influence of structural modifications at the 3 ' and 4' positions of doxorubicin on the drug ability to trap topoisomerase II and to overcome multidrug resistance. Mol. Pharmacol.. 45, 908-915.

4. Cardoso F., Durbecq V., Larsimont D., Paesmans M., Leroy J. Y., Rouas G., Sotiriou C., Renard N., Richard V., Piccart M. J. and Di Leo A. (2004): Correlation between complete response to anthracycline-based chemotherapy and topoisomerase II-alpha gene amplification and protein overexpression in locally advanced/metastatic breast cancer. Int. J. Oncol., 24, 201-209.

5. De Boer R. A., van Veldhuisen D. J., van der Wijk J., Brouwer R. M., de Jonge N., Cole G. M. and Suurmeijer A. J. (2000): Additional use of immunostaining for active caspase 3 and cleaved actin and PARP fragments to detect apoptosis in patient with chronic heart failure. J. Card. Fail., 6, 330-337.

6. Den Boer M. L., Pieters R. and Veerman A. J. (1998): Mechanisms of cellular anthracycline resistance in childhood acute leukemia. Leukemia, 12, 1657-1670.

7. De Graff W. G., Myers L. S. Jr., Mitchell J. B. and Hahn S. M. (2003): Protection against adriamycin cytotoxicity and inhibition of DNA topoisomerase II activity by 3,4-dihydroxybenzoic acid. Int. J. Oncol., 23, 159-163.

8. Di Leo A. and Isola J. (2003): Topoisomerase II alpha as a marker predicting the efficacy of anthracyclines in breast cancer: are we at the end of the beginning? Clin. Breast Cancer, 4, 179-186.

9. Durbecq V., Paesmans M., Cardoso F., Desmedt C., Di Leo A., Chan S., Friedrichs K., Pinter T., Van Belle S., Murray E., Bodrogi I., Walpole E., Lesperance B., Korec S. Crown J., Simmonds P., Perren T. J., Leroy J. Y., Rouas G., Sotiriou C., Piccart M. and Larsimont D. (2004): Topoisomerase II-alpha expression as a predictive marker in a population of advanced breast cancer patients ran- domly treated either with single-agent doxorubicin or single-agent docetaxel. Mol. Cancer Ther., 3, 1207-1214.

10. Gariboldi M. B., Ravizza R., Riganti L., Meschini S., Calcabrini A., Marra M., Arancia G., Dolfini E. and Montie E. (2003): Molecular determinants of intrinsic resistance to doxorubicin in human cancer cell lines. Int. J. Oncol., 22, 1057-1064.

11. Gewirtz D. A. (1999): A critical evaluation of the mechanism of action proposed for the antitumor effects of the anthracycline antibiotics adriamycin and daunorubicin. Biochem. Pharmacol., 57, 727-741.

12. Grabowski D. R., Dubyak G. R., Rybicki L., Hidaka H. and Ganapathi R. (1998): Tumor cell resistance to topoisomerase II poisons. Role for intracellular free calcium in the sensitization by inhibitors of calcium-calmodulin-dependent enzymes. Biochem. Pharmacol., 56, 345-349.

13. Gruber B. M., Anuszewska E. L., Bubko I., Goździk A., Priebe W. and Fokt I. (2005): Relationship between topoisomerase II-DNA cleavable complexes, apoptosis and cytotoxic activity of anthracyclines in human cervix carcinoma cells. Anticancer Res., 25, 2193-2198.

14. Gruber B. M., Anuszewska E. L. and Priebe W. (2004): The effect of new anthracycline derivatives on the induction of apoptotic processes in human neoplastic cells. Folia Histochem. Cytobiol., 42, 127-130.

15. Gruber B. M., Anuszewska E. L., Roman I., Goździk A., Priebe W. and Fokt I. (2006): Topoisomerase II alpha expression and cytotoxicity of anthracyclines in human neoplastic cells. Acta Pol. Pharm. Drug Res., 63,15-18.

16. Jarvinen T. A. and Liu E. T. (2003): HER-2/neu and topoisomerase II alpha in breast cancer. Breast Cancer Res. Treat., 78, 299-311.

17. Koshiyama M., Fujii H., Kinezaki M. and Yoshida M. (2001): Correlation between topo II alpha expression and chemosensitivity testing for topo II-targeting drugs in gynaecological carcinomas. Anticancer Res., 21, 905-910.

18. Lage H. and Dietel M. (2002): Multiple mechanisms confer different drug resistant phenotypes in pancreatic carcinoma cells. J. Cancer Res. Clin Oncol., 128, 349-357.

19. Li T.-K. and Liu L. F. (2001): Tumor cell death induced by topoisomerase-targeting drugs. Annu. Rev. Pharmacol. Toxicol., 41, 53-77.

20. MacGrogan G., Rudolph P., Mascarel Id I., Mauriac L., Durand M.,Avril A., Dilhuydy J. M., RobertJ., Mathoulin-Pelissier S., Picot V., Floquet A., Sierankowski G. and Coindre J. M. (2003): DNA topoisomerase II alpha expression and the response to primary chemotherapy in breast cancer. Br. J. Cancer, 89, 666-671.

21. Martin-Richard M., Munoz M., Albanell J., Colomo L., Bellet M., Rey M. J., Tabernero J., Alonso C., Cardesa A., Gascon P. and Fernandez P. L. (2004): Serial topoisomerase II expression in primary breast cancer and response to neoadjuvant anthracycline-based chemotherapy. Oncology, 66, 388-394.

22. Nowak R. and Tarasiuk J. (2004): The inhibition of apoptosis in cancer cells resistant to anticancer drugs (in Polish). Postępy Biochem., 50, 330-341.

23. Priebe W. (1995): Mechanism of action-governed design of anthracycline antibiotics: a "turn-off/ turn-on" approach. Curr. Pharm. Des., 1, 51-68.

24. Ramachandran Ch., Samy T. S., Huang X. L., Yuan Z. K. and Krishan A. (1993): Doxorubicin-induced DNA breaks, topoisomerase II activity and gene expression in human melanoma cells. Biochem. Pharmacol., 45, 1367-1371. 
25. Sehested M. and Jensen P. B. (1996): Mapping of DNA topoisomerase II poisons (etoposide, clerocidin) and catalytic inhibitors (aclarubicin, ICRF-17) to four distinct steps in the topoisomerase II catalytic cycle. Biochem. Pharmacol., 51, 879-886.

26. Trevino A. V., Woynarowska B. A., Herman T. S., Priebe W. and Woynarowski J. M. (2004): Enhanced topoisomerase II targeting by annamycin and related 4-deme- thoxy anthracycline analogues. Mol. Cancer Ther., 3, 1403-1410.

27. Yamazaki K., Isobe H., Hanada T., Betsuyaku T., Hasegawa A., Hizawa N., Ogura S. and Kawakami Y. (1997): Topoisomerase II alpha content and topoisomerase II catalytic activity cannot explain drug sensitivities to topoisomerase II inhibitors in lung cancer cell lines. Cancer Chemother. Pharmacol., 39, 192-198. 